\title{
Labyrinthe
}

$35 \mid 2010$ (2)

Empire Reader

\section{Comment les empires touchent à leur fin}

\section{Charles Tilly}

Traducteur : Marc Aymes

\section{OpenEdition}

Journals

Édition électronique

URL : https://journals.openedition.org/labyrinthe/4077

DOI : $10.4000 /$ labyrinthe.4077

ISSN : 1950-6031

Éditeur

Hermann

Édition imprimée

Date de publication : 28 août 2010

Pagination : 21-37

ISBN : 9782705669973

Référence électronique

Charles Tilly, "Comment les empires touchent à leur fin », Labyrinthe [En ligne], 35 | 2010 (2), mis en ligne le 27 juillet 2012, consulté le 16 février 2023. URL : http://journals.openedition.org/labyrinthe/ 4077 ; DOI : https://doi.org/10.4000/labyrinthe.4077 


\section{Comment les empires touchent à leur fin}

Charles TILLY

(C) 1997 Westview Press. La version originale de ce texte est parue dans Karen Barkey, Mark von Hagen (dir.), After Empire : Multiethnic societies and nationbuilding. The Soviet Union, and the Russian, Ottoman, and Habsburg empires, Boulder, Westview Press, 1997, p. 1-11. Reproduction sur autorisation.

\begin{abstract}
D'Hérodote à Montesquieu et au-delà, les poètes, historiens et philosophes se sont adonnés sans relâche à ce qui, dans notre culture, constitue l'une des formes littéraires consacrées : le
\end{abstract} requiem pour un empire défunt. En tant qu'objet de réflexion, le déclin des empires est de ceux qui résonnent à l'échelle de l'histoire globale, car il signale à tous combien sont faillibles ces entreprises humaines qui semblaient si inébranlables. La stupeur de voir s'effondrer ce qui autrefois fut grand crée un
" Ici, me dis-je, ici fleurit jadis une ville opulente ici fut le siège d'un empire puissant. Oui ! ces lieux maintenant si déserts, jadis une multitude vivante animait leur enceinte ; une foule active circulait dans ces routes aujourd'hui solitaires. [...] Et maintenant voilà ce qui subsiste de cette ville puissante, un lugubre squelette ! Voilà ce qui reste d'une vaste domination, un souvenir obscur et vain ! [...] Ah ! comment s' est éclipsée tant de gloire ! Comment se sont anéantis tant de travaux !... Ainsi donc périssent les ouvrages des hommes ! ainsi s'évanouissent les empires et les nations ! »

C. F. Volney, Les Ruines, ou Méditation sur les révolutions des empires, Paris, Parmantier I Froment, 1826 [1791], p. 5-6.

effet de contraste, dont se nourrit tout un texte de méditations morales sur le déclin impérial et d'oraisons déclamées parmi les ruines. Ce texte a inspiré aussi bien les conquérants nouveaux venus, clamant leur supériorité sur les vaincus, que les philosophes soucieux de mettre en garde contre les excès de la démesure. (Nous autres qui, aujourd'hui, glosons sur l'effondrement de l'empire soviétique, devrions nous demander dans quelle catégorie nous sommes.) Au titre des déplorations philosophiques, il n'est que de rappeler les fameuses lignes que Lewis Mumford consacra à Rome :

L'histoire de Rome indique, avec un relief particulier, ce qui, dans le domaine politique aussi bien que dans celui de l'urbanisme, doit être à tout prix évité. Nous voyons là de multiples signaux d'alarme, indiquant 
le départ de pistes dangereuses. Lorsque, dans des centres surpeuplés, les conditions d'habitat se détériorent, tandis que le prix des loyers monte en flèche, lorsque le souci d'exploiter de lointains territoires vient pallier les carences de l'harmonie interne, nous songeons inévitablement au précédent de ce que Rome avait construit. Ainsi retrouvons-nous aujourd'hui les arènes, les immeubles de rapport, les exhibitions, les grands spectacles, avec nos matchs de football, nos concours de beauté, le continuel strip-tease que répètent les motifs des panneaux réclames, cette constante titillation des sens par l'érotisme, la boisson, la violence, dans un climat digne en tout point de la Rome antique. Sans compter la multiplication des salles d'eaux, la sur-dépense des moteurs sur le bitume des larges autostrades, et couronnant le tout, l'accumulation collective effrénée de fugacités enjôleuses de toutes sortes, merveilles d'audace technique. Lorsqu'enfle ainsi un pouvoir moralement abattu, tandis que la vie s'étiole, ce sont là les symptômes de la fin. L'édifice est encore solide et pas une pierre n'a bougé, mais ces signes ne trompent pas: les Barbares se sont infiltrés dans les défenses, ils sont installés dans nos murs. Ces signes sont ceux de la prochaine nécropole. Le bourreau attend. Paraîtront bientôt les vautours ${ }^{1}$.

C'est là pour Mumford un moyen de faire valoir sa théorie du pouvoir politique et de la virtuosité technique : si leur croissance passe les bornes de la sobriété, la vie s'en trouve déshumanisée, et leur propre anéantissement précipité. Dans une veine similaire, quoique moins pompeusement, Alex Motyl a souligné que « l'absolutisme engendre des pathologies qui aboutissent à sa propre dégénérescence, ce qui, au sein d'un empire à territoires contigus, provoque la détérioration du contrôle exercé par le centre sur la périphérie ${ }^{2} »$.

Cela dit, avant d'entreprendre de savantes autopsies, mieux vaudrait s'assurer de ce que l'organisme fût malade, et effectivement trépassé. Après tout, les empires n'ont-ils pas été la forme étatique dominante et la plus étendue, depuis que le monde a vu se constituer des États solidement établis ? Rien, sinon une catastrophe tellurique, ne semble à même d'éradiquer ces dinosaures carnassiers. C'est aujourd'hui seulement, au vingtième siècle, que nous semblons laisser derrière nous l'ère des immenses empires eurasiens, entamée quelque quatre mille ans auparavant dans l'immense région s'étendant de la Méditerranée à l'Asie orientale. Il n'est pas exclu que des accords internationaux comme l'Union européenne, le GATT et l'ALÉNA, puissent être considérés 


\section{Comment les empires touchent à leur fin}

comme des projets impériaux. Bref, le requiem entonné de nos jours pourrait se révéler prématuré.

Et à supposer que les empires soient bel et bien en train de disparaître, leur fin demeure aussi difficile à comprendre que l'extinction subite des dinosaures. Le siècle qui s'achève compte parmi les plus sanglants et les plus militaires au monde : la désintégration des empires signifie-t-elle que les hostilités conquérantes entre États vont également décliner, pour mieux céder la place à la guerre civile et au génocide ? La dispersion des empires d'autrefois, y compris par la décolonisation de grande ampleur survenue à partir des années 1960 , préfigure-t-elle le sort que connaîtront les débris des dislocations plus récentes? La fin des empires enfante-t-elle de nouvelles formes de conflit, interne ou externe ? Où et quand un tel phénomène survient-il, et dans quelle mesure se généraliset-il ? La dissolution du contrôle central s'accompagne-t-elle d'explosions de nationalisme, au nom des fragments de l'ancien empire ? À quelles conditions les États successeurs constituent-ils - ou, en l'occurrence, seraient-ils à même de constituer — des régimes démocratiques stables ? Que l'histoire impériale ait atteint son terme ou non, les cycles de déclin antérieurs nous livrent de quoi nous interroger, et quantité de matière à comparer.

Pour qui se lance dans cette démarche comparatiste, il importe d'éviter toute arrogance conjecturale : les empires ne périssent pas simplement d'avoir adopté des formes de gouvernement non viables. Ce sont des bêtes robustes, l'histoire en témoigne. L'empire chinois, sous différentes variantes, a perduré deux millénaires ou plus ; l'empire byzantin s'est poursuivi plus de mille ans ; l'empire romain a duré six siècles ; l'empire ottoman a survécu près d'un demi-millénaire ; divers empires mongols ont occupé durant cinq cents ans une surface de territoires contigus plus vaste qu'aucune autre organisation politique ne le fit jamais ; et quant aux plus brefs, mais essentiels, empires britannique, français, allemand, italien, espagnol, portugais, belge, hollandais, américain, russe, soviétique et austro-hongrois, leur dénouement demeure présent à la mémoire de nos contemporains.

Rien qu'en Europe, dans l'intervalle entre les poids lourds romain et britannique, les grands empires furent légion : normand, lituanien-polonais, suédois, bourguignon, et beaucoup d'autres encore. La domination du continent par des États consolidés ne vint qu'ensuite. Autour de la Méditerranée, la structure des États musulmans les plus importants fut 
impériale. Et pendant ce temps, en Amérique du Sud, en Afrique, en Asie du Sud-Est, d'autres empires encore connaissaient grandeur et décadence. Aussi le fait impérial n'est-il, dans notre monde à nous, que de souche récente : raison pour laquelle, à disserter sur la fin des empires en termes généraux et définitifs, nous risquons l'erreur chinoise typique, celle de confondre le déclin d'un régime particulier avec l'arrivée à son terme ultime d'une forme politique auparavant dominante. De même qu'il serait inconsidéré d'exulter bruyamment de la « démocratisation » irréversible d'un monde où la
« L'Empire n'est guère pensé, et peut-être guère pensable au sein de la tradition occidentale, c'està-dire dans les limites de la métaphysique de la subjectivité. Tout au plus a-t-on pu y penser le dépassement de l'État moderne sur son propre terrain ; et cela a donné les irrespirables projets d'État universel, les spéculations sur le droit cosmopolite qui viendrait finalement instaurer la paix perpétuelle ou encore le ridicule espoir d'un État démocratique mondial, qui est la perspective ultime du négrisme. [...] Les Chinois, qui ont pris leurs quartiers hors de la métaphysique de la subjectivité entre le vi $l^{e}$ et le \|\|$^{e}$ siècle avant notre ère, se forgèrent alors une théorie de la souveraineté impersonnelle qui n'est pas sans utilité pour comprendre les ressorts actuels de la domination impériale ».

Tiqqun, Contributions à la guerre en cours, Paris, La Fabrique, 2009 [2001], p. 83-84 et 88. guérilla, le génocide et le politicide tendent à devenir choses communes ${ }^{3}$, gardons-nous de déclarer tout à trac : c'en est fait des empires, ils ont à jamais rejoint le pays de leurs ancêtres.

Les traces dont nous disposons concernant l'existence d'États remontent, pour les plus anciennes, à environ dix millénaires. Sur cette période, trois formes étatiques principales se sont imposées : la cité-État, le domaine militaro-agraire et l'empire — ou diverses combinaisons des trois, à l'instar de Venise, cité-État s'adjoignant un empire maritime disséminé, ou de la République hollandaise, fédération malaisée de cités-États. Les États consolidés — organisations coercitives gouvernant de manière directe et relativement uniforme un ensemble de territoires hétérogènes clairement

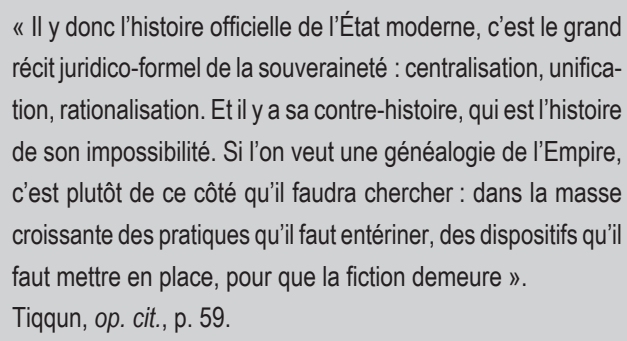
récit juridico-formel de la souveraineté : centralisation, unification, rationalisation. Et il y a sa contre-histoire, qui est l'histoire de son impossibilité. Si l'on veut une généalogie de l'Empire, c'est plutôt de ce côté qu'il faudra chercher : dans la masse croissante des pratiques qu'il faut entériner, des dispositifs qu'il faut mettre en place, pour que la fiction demeure ». Tiqqun, op. cit., p. 59 . 
délimités - ne se sont imposés comme forme étatique dominante qu'au cours deux derniers siècles, d'abord dans le monde européen puis, par la conquête et l'émulation, sur l'ensemble du globe.

N'allons pas non plus en déduire que ces États consolidés aient présenté de tels avantages sur les autres organisations politiques qu'ils les aient rendues obsolètes. Après seulement deux siècles d'hégémonie, les États consolidés d'Occident laissent déjà voir des signes de leur impuissance à assurer l'ordre ou à fournir des biens publics, défiés qu'ils sont par les réseaux du capital et du travail, du trafic de drogue ou d'armes, de la terreur, qui tous franchissent avec aisance leurs frontières péniblement érigées ${ }^{4}$. D'ici un siècle, il se pourrait que les États consolidés ne soient plus considérés par les analystes que comme un phénomène éphémère, tandis que les empires demeureraient à leurs yeux les formes historiquement dominantes d'organisation politique à l'échelle supra-régionale.

Un empire est une entité politique [polity] vaste et composite, liée à une autorité centrale par un système de gouvernement indirect. L'autorité centrale exerce une certaine faculté de contrôle militaire et fiscal dans chaque région essentielle de son domaine impérial, mais tolère deux modalités majeures de médiation : (1) la rétention ou l'institution, pour le gouvernement de chaque région, de conventions particulières et distinctes; (2) l'exercice du pouvoir par des intermédiaires jouissant d'une autonomie régionale considérable, en contrepartie de laquelle ils doivent allégeance, versement d'un tribut et acquittement d'un service militaire au centre.
« Le Système avait alimenté le marché international de l'habillement, royaume de l'élégance italienne. Chaque recoin de la planète pouvait être atteint par les entreprises, les hommes et les produits du Système. Système: un mot qu'ici tout le monde connaît mais qui, pour les autres, reste encore à déchiffrer, une référence inaccessible à ceux qui ignorent quelles sont les dynamiques du pouvoir de l'économie criminelle. Le mot camorra n'existe pas, c'est un mot de flics, utilisé par les magistrats, les journalistes et les scénaristes. Un mot qui fait sourire les affiliés, une indication vague, un terme bon pour les universitaires et appartenant à l'histoire ".

Roberto Saviano, Gomorra. Dans l'empire de la camorra (trad. de l'italien par Vincent Raynaud), Paris, Gallimard, Folio, 2009 [2006], p. 67. 
Nul doute que les étudiants de Suisse, des États-Unis, de République fédérale d'Allemagne, du Brésil ou d'Afrique du Sud ne s'empressent de reconnaître en ces critères des différences de degré, plutôt que des distinctions absolues; et cependant, au sein de leurs sphères de compétence, les gouvernements fédéraux actuels règnent autrement plus uniformément et directement que ne le faisaient les États ottoman ou mongol. La raison pour laquelle les empires gouvernent indirectement suivant une géométrie de conventions variable, c'est qu'ils s'établissent et s'accroissent par la conquête militaire d'entités existantes, avec la collaboration (sous contrainte ou non) d'hommes de pouvoir locaux qui conservent une substantielle capacité discrétionnaire dans leur juridiction. Cette logique a pour réciproque que, lorsque ces empires se désintègrent, cela résulte ordinairement d'une combinaison de conquêtes exogènes et de résistances périphériques, dont les principaux protagonistes sont souvent des agents anciennement au service du centre.

L'empire, en tant que forme de gouvernement à grande échelle, s'est révélé particulièrement récurrent et adaptable, et ce pour deux raisons étroitement corrélées : il fait tenir ensemble des unités disparates de plus petite taille, sans que le centre ait à leur imposer tellement de transformations internes ; il permet aux gouvernants de se procurer des ressources sans que ce pompage soit coûteux en termes de surveillance et de répression. Afin d'extraire le minimum requis de tribut, de soutien militaire et de loyauté devant revenir au centre, les dirigeants régionaux mobilisent les pratiques, les ententes et les liens déjà en vigueur. Cela leur permet d'obtenir autant de revenus et de services que possible, sans avoir à calculer ce que la région peut effectivement fournir au niveau global, local ou individuel. Tant qu'ils disposent de forces pouvant être déployées à leur main, et qu'ils ont l'oreille des autorités impériales en cas d'urgence, point n'est besoin pour le centre d'établir un système dense de maintien de l'ordre dans les régions, et l'instauration de mécanismes de contrôle aux frontières, qu'exigerait la perception finement tamisée d'impôts personnels et fonciers ou de droits d'accise, s'impose encore moins $^{5}$. Nul doute que l'extraction impériale des ressources s'effectue généralement au prix de considérables déperditions, fraudes, connivences personnelles et inégalités - et pour cette raison, lorsqu'un empereur exige soudain des revenus supplémentaires (ou, à l'inverse, se voit ouvertement privé d'une part de son pouvoir coercitif), des sujets auparavant soumis en viennent fréquemment à se rebeller. Mais précisément, la simplicité 


\section{Comment les empires touchent à leur fin}

sans fioritures du système impérial est ce qui le rend adaptable à toutes sortes de terrains sociaux.

Il arrive par conséquent que l'expansion d'un empire soit d'une rapidité saisissante, loin de la lente accrétion d'une cité-État, d'un domaine agraire ou d'un État consolidé : c'est que la conquête militaire s'y combine avec une politique de cooptation, absorbant les systèmes de pouvoir en place dans ses réseaux de tributaires et d'alliés militaires. Ainsi eut lieu la prompte montée en puissance des prédateurs impériaux perse, mongol et ottoman - tous ayant pour cheville ouvrière, à l'origine, une cavalerie armée - aux portes de leurs voisins agraires. Mais pour les mêmes raisons, la chute d'un empire peut être tout aussi spectaculaire. Plusieurs causes peuvent expliquer cette ruine précipitée : (1) les entités politiques placées sous tutelle impériale peuvent aisément s'en détacher, eu égard à leur faible intégration au sein d'un quelconque réseau administratif ; (2) le pouvoir autonome dont jouissent les vice-rois inclut celui de faire défection ; (3) les populations sujettes demeurent attachées à leur caractère distinct en matière d'identité, de mémoire ou de rancune ; et (4) lorsque le centre devient vulnérable, la nouvelle se répand vite parmi les sujets de l'empire et ses voisins hostiles. Si l'empire chinois s'est révélé plus durable que les autres, c'est qu'il sut parer à ces quatre périls simultanément : il déployait une structure administrative relativement uniforme jusqu'au niveau le plus local, intégrant les hobereaux à un système de compétition pour l'obtention de faveurs impériales ; il mutait fréquemment les agents de sa bureaucratie, en interdisant leur affectation dans leur province d'origine ; il stimulait la mobilité interne et minimisait l'importance des distinctions ethniques ; il n'hésitait pas à faire montre de sa force partout sur son vaste territoire ; et ce faisant, l'État impérial fut reconduit, dynastie après dynastie, siècle après siècle, de conquête en revers et de revers en conquête ${ }^{6}$.

C'est pourquoi le scepticisme s'impose face aux analyses qui expliquent la faillite du communisme en affligeant sa structure impériale d'un talon d'Achille unique. Il en va ainsi, entre autres, de la notion d' « absolutisme » invoquée par Alex Motyl. Semblable interprétation ne quitte guère les sentiers battus d'une approche internaliste et universelle. Internaliste, car elle situe les origines de l'effondrement au sein même du système. Universelle, en ce qu'elle prétend expliquer pareillement la chute de tout système analogue. Également internaliste et universelle est, à cet égard, l'analyse de Joseph Tainter, qui impute l'« écroulement » 
à la décroissance des rendements marginaux perçus par l'unité centrale de contrôle? .

Nombre d'autres interprétations, à l'inverse, mettent l'accent sur l'unicité de leur(s) cas d'étude, et/ou sur l'externalité des ressorts explicatifs. L'approche la plus courante est encore celle qui, tout en invoquant à la fois des causes uniques et générales pour comprendre un déclin donné, les associe à une analyse équilibrée des facteurs internes et externes ${ }^{8}$. Le propos internaliste-universaliste de Motyl, quant à lui, postule l'existence d'un processus récurrent : il suppose en tout empire à territoires contigus une caractéristique nécessaire, l'absolutisme, qui sape les conditions de sa propre survie. Convient-il vraiment de parler d' " absolutisme » pour décrire un mode opératoire de l'État quel qu'il soit, plutôt que de réserver le terme à la revendication par un souverain d'une primauté bénie des cieux censée le distinguer des magnats régionaux ${ }^{9}$ ? Point n'est besoin d'insister sur le caractère douteux d'un tel usage. Passons aussi sur le choix par Motyl du mot «préfet », quintessence du volontarisme centralisateur, pour désigner les intermédiaires à demi autonomes qui se mettent au service des régimes impériax ${ }^{10}$.

L'idée essentielle, en l'espèce, fait écho à la caractérisation classique des empires - sorte de théorie du problème du principal-agent avant la lettre $^{11}$ - qu' avait proposée Shmuel Eisenstadt ${ }^{12}$. Le problème auquel tout empire fait face, en effet, est de s'assurer un assentiment soutenu, ainsi que des sources d'information dignes de confiance, parmi les agents régionaux. La chose est d'autant moins aisément acquise que ces derniers sont prompts à développer des liens, des intérêts et des aptitudes qui les incitent à subvertir l'entreprise impériale, à faire alliance avec ses ennemis, ou même à entrer en rébellion de leur propre chef. Supposer, cependant, que tout empire succombe des suites de ce dilemme, c'est tirer un trait sur l'immense variabilité des durées et des fins d'empire, et oublier le rôle crucial que, de manière récur-

« Je ne crois pas à “l'admirable construction de l'ordre romain". Rome est un empire dont l'administration est aussi sommaire qu'archaïque : de science du gouvernement, elle n'en a pas ; elle a une science de la puissance et un culte de sa puissance. [...] Ce n'est pas un ordre, c'est une conjonction de chances et de facteurs négatifs. Tout cela est plat comme la main $»$.

Paul Veyne, Le Quotidien et l'intéressant. Entretien avec C. DarboPeschanski, Paris, Les Belles Lettres, 1995, p. 96-97. 


\section{Comment les empires touchent à leur fin}

« Le plus grand fleuve du monde, le Mississippi, Père des Eaux, fut le digne théâtre de cette incomparable canaille. (Ce fut Alvarez de Pineda qui découvrit le Mississippi et le premier qui l'explora fut le capitaine Hernando de Soto, ancien conquérant du Pérou, qui adoucit les mois de captivité de l'Inca Atahualpa en lui apprenant à jouer aux échecs ; quand il mourut, on lui donna pour sépulture les eaux du fleuve.)

Le Mississippi est un fleuve aux larges épaules. C'est le frère sombre et infini du Parana, de l'Uruguay, de l'Amazone et de l'Orénoque. C'est un fleuve aux eaux mulâtres. Plus de quatre cents millions de tonnes de boue insultent annuellement le golfe du Mexique où il les déverse. Une telle masse de résidus anciens et vénérables a formé un delta où les gigantesques cyprès des marais vivent des dépouilles d'un continent en perpétuelle dissolution, où les labyrinthes de boue, de poissons morts et de joncs reculent les frontières et assurent la paix de ce fétide empire ».

Jorge Luis Borges, « Le rédempteur effroyable Lazarus Morrell », Histoire universelle de l'infamie, CEuvres complètes, Paris, NRF-Gallimard, vol. I, 1993 [1935], p. 304. rente, jouent les conquérants venus d'ailleurs.

Comment les empires touchent-ils à leur fin ? Autant se demander comment les fleuves changent de lit, comment les barrières de corail périssent, comment les lignées humaines s'éteignent. In vitro, il pourrait sembler possible de tracer un cours unique suivi par tous, empires, lignées, fleuves, coraux. Mais une vérification in vivo confirme qu'il n'en va pas ainsi. L'histoire des empires, quatre millénaires de prévalence et de variété, ne se laisse pas réduire à des constantes si ce n'est à celles, triviales, que je citais plus haut : dans toute chute, il entre une part de conquête depuis l'extérieur, et une part de défection à l'intérieur. Soit le cas de l'Union soviétique : à la rigueur, nous pourrions généraliser en insistant sur l'épuisement de sa force et de sa crédibilité militaires durant la guerre d'Afghanistan, sur les répercussions politiques de la non-intervention gorbatchévienne dans les États du Pacte de Varsovie, sur le champ d'attraction établi par le monde capitaliste via les pays baltes. Mais si l'objectif était d'analyser systématiquement ce que l'expérience soviétique partage avec
« L'émergence effective d'un empire, à un moment donné du temps, n'est jamais acquise, tant est grande la part du hasard dans le processus. Un modèle de prédiction ne peut donc être que stochastique, non déterministe ».

Peter Turchin, "A theory for formation of large empires », Journal of Global History, 4, 2009, p. 200. 
«Personne ne résiste aux raz de marée ni aux volcans. Il y a lieu de craindre, dans l'incertitude des années qui viennent, la surrection de raz de marée humains, d'éruptions populaires de types volcaniques, de tornades, d'incendies et de désastres. Peut-être en naîtront des empires, pour le bonheur et surtout pour le malheur des peuples. Nés dans la tourmente, eux aussi ils périront un jour ».

Jean-Baptiste Duroselle, Tout Empire périra. Théorie des relations internationales, Paris, Armand Colin, 1992 [1981], p. 295-296. celle d'autres empires, nous n'aurions guère avancé dans cette voie. Loin de moi l'idée de bouder le plaisir qu'il y aurait à comparer l'effondrement soviétique et la dissolution de l'empire des Habsbourg. Mais soyons avertis : la recherche de ressemblances trait pour trait

restera, fondamentalement, de peu d'utilité. Nous gagnerons davantage à resituer les empires et leurs fins dans un champ de signification à géométrie variable.

Au demeurant, Alex Motyl a amorcé cette démarche essentielle de différenciation, en proposant de distinguer les empires suivant que leurs territoires sont contigus ou au contraire disséminés. Pour autant, cette distinction n'offre pas de solution évidente à nos problèmes. Ainsi, devrait-on considérer le réseau maritime disséminé de l'empire vénitien comme moins solide que celui, contigu mais essentiellement terrestre, de l'empire russe ? ou encore supposer que la suffocation de Venise par l'expansion ottomane diffère fondamentalement de la débâcle byzantine, précipitée par l'entente vénéto-ottomane peu auparavant ${ }^{13}$ ? Il n'y a rien d'évident à cela, tant, jusqu' au siècle dernier, le voyage par mer l'emportait sur tout autre mode de transport. Plus probablement, cette dimension de la variation requiert une analyse en termes de coûts de transaction. Grosso modo, les hypothèses seraient les suivantes : (1) plus le maintien du contrôle central sur un segment impérial a un coût élevé, plus il devient probable que ce segment se détache de l'empire ; et (2) plus les coûts de transaction totaux supportés par l'empire augmentent relativement aux revenus escomptés de l'ensemble des segments, plus les risques de désintégration sont aigus.

Faute de disposer d'étalons de mesure bien établis pour estimer les coûts de transactions et les revenus de l'empire, il est à prévoir que cette formulation en termes de coût-avantage instille à notre raisonnement une forme de circularité. Elle n'en permet pas moins d'évaluer les effets produits par des changements significatifs, comme lorsque le flux de métaux précieux 


\section{Comment les empires touchent à leur fin}

d'Amérique du Sud vers l'Espagne s'interrompt, ou lorsque les pirates pullulent le long des routes marchandes d'un empire maritime ${ }^{14}$. Que des sources essentielles de revenus se tarissent, ou que les coûts induits par le contrôle s' accroissent, et aussitôt l'empire perd en espérance de vie. Si ses lignes de transmission et d'approvisionnement s'allongent à l'excès, nul doute que sa vulnérabilité à ces deux formes de perturbation augmente. Reposée ainsi, la distinction qu'a introduite Motyl permet d'identifier une dimen-

\begin{abstract}
"Toute existence a son apogée, une époque pendant laquelle les causes agissent et sont en rapport exact avec les résultats. Ce midi de la vie, où les forces vives s'équilibrent et se produisent dans tout leur éclat, est non seulement commun aux êtres organisés, mais encore aux cités, aux nations, aux idées, aux institutions, aux commerces, aux entreprises qui, semblables aux races nobles et aux dynasties, naissent, s'élèvent et tombent. D'où vient la rigueur avec laquelle ce thème de croissance et de décroissance s'applique à tout ce qui s'organise ici-bas ? [...] L'Histoire, en redisant les causes de la grandeur et de la décadence de tout ce qui fut ici-bas, pourrait avertir l'homme du moment où il doit arrêter le jeu de toutes ses facultés ; mais ni les conquérants, ni les acteurs, ni les femmes, ni les auteurs n'en écoutent la voix salutaire. [...] Pourquoi de nouvelles pyramides ne rappelleraient-elles pas incessamment ce principe qui doit dominer la politique des nations aussi bien que celle des particuliers : Quand l'effet produit n'est plus en rapport direct ni en proportion égale avec sa cause, la désorganisation commence? » Honoré de Balzac, Histoire de la grandeur et de la décadence de César Birotteau, Paris, Pocket, 1994 [1837], p. 69-70.
\end{abstract}

sion essentielle de la variation impériale.

De quels autres aspects devrions-nous souligner l'importance? Plusieurs candidats peuvent être pressentis. On l'a dit, les empires ont pour caractéristique d'être des entités politiques vastes, composites, et liées à des autorités centrales par un système de gouvernement indirect : la taille ne saurait donc être quantité négligeable, pas plus que la structure militaire et technologique, les relations entre centre impérial et puissances extérieures, l'organisation des relations internationales, le degré d'hétérogénéité entre les régions gouvernées, l'ampleur des inégalités entre centre et périphérie, les mécanismes de gouvernement direct, et, surtout, la structure économique, politique et sociale du centre lui-même. L'ensemble de ces caractéristiques, tout ou partie, forme un champ de variation systématique au sein duquel, en principe, s'inscrivent la survie, la mutation ou l'effondrement d'un empire, processus de transformation et retombées de dislocation compris. Cela n'aide guère, certes ; mais au moins, nous disposons d'un pense-bête. 
« Pour ce qui est de l'Histoire... [...] Aujourd'hui les choses vont mal. La Russie est en train de s'emparer de la planète ; l'Amérique, empêtrée par la superstition de la démocratie, ne se résout pas à être un empire. De jour en jour notre pays devient plus provincial. Plus provincial et plus suffisant, comme s'il refusait de voir. Je ne serais guère surpris que l'enseignement du latin soit remplacé par celui du guarani ».

Jorge Luis Borges, "L'autre », Le Livre de sable, CEuvres complètes, Paris, NRF-Gallimard, vol. II, 1999 [1975], p. 484.
Peut-être vaudrait-il mieux adopter une procédure inverse, si nous voulons aller plus loin : partir des retombées présumées des transformations impériales, pour remonter ensuite aux interprétations de leurs régularités. Par exemple, dans quelles conditions et suivant quelles trajectoires pourrait-on s'attendre à ce que des empires en voie d'effondrement ouvrent la voie à des systèmes politiques

démocratiques ? Dans le débat actuel sur ces questions, la démocratie est généralement définie de manière substantive et procédurale. Substantive, car on s'intéresse principalement aux résultats, comme l'égalité ou l'harmonie ; procédurale, car l'accent porte sur certains dispositifs, comme les élections libres ou les passations de pouvoir ${ }^{15}$. Pour ma part, je m'en tiens à une conception « institutionnelle » vieux jeu de la démocratie, centrée sur les relations entre les citoyens et les États. Elle s'appuie sur un ensemble de concepts qu'il me faut, hélas, définir pour éviter toute confusion :

- État : organisation qui, au sein d'un territoire délimité, contrôle l'essentiel des moyens de coercition concentrés, et jouit à certains égards de la primauté vis-à-vis de toutes les autres organisations existantes sur le même territoire.

- Régime politique [polity] : jeu de relations établies entre les agents de l'État et l'ensemble des acteurs politiques majeurs sur le territoire.

- Droits : ensemble de réclamations susceptibles d'application ; l'inverse des obligations.

- Citoyenneté : droits et obligations mutuels liant les agents d'un État et une catégorie de personnes établie sur la seule base de leurs attaches juridiques au même État.

Aux termes de cette conception, un régime politique est démocratique dans la mesure où il établit (1) une citoyenneté élargie, (2) l'égalité des citoyens, (3) la nécessité que ceux-ci soient consultés pour déterminer qui gouverne et comment, et (4) leur protection à l'égard de tout arbitraire étatique. À cette aune, aucun régime politique n'est pleinement démocratique : dans 


\section{Comment les empires touchent à leur fin}

la réalité, on oscille entre le franchement non-démocratique (une citoyenneté inégale et étriquée, sans nécessité de consultation ou de protection) et le relativement démocratique (résultats élevés sur les quatre indices de valeur).

Suivant ces critères, quand bien même des segments d'empires pourraient-ils en principe connaître une situation un tant soit peu démocratique, les empires dans leur entier sont et demeurent, par définition, non-démocratiques. La segmentation et le recours au gouvernement indirect qui les caractérisent, s'ils n'empêchent nullement la formation d'un vaste corps politique, constituent à l'échelle impériale une entrave à l'instauration de citoyens égaux entre eux, consultés et protégés. Partant, notre effort de définition permet de mettre en lumière ce qu'implique une transition impériale vers la démocratie : ou
« Ce n'est ni à un champ pétrolifère, ni à un jardin, que ressemble la démocratie : c'est à un lac. La formation du lac — grande masse aquifère situé à l'intérieur des terres — peut advenir lorsqu'un torrent de montagne débouche sur un bassin naturel préexistant ; lorsque quelqu'un, ou quelque chose, obstrue le cours d'un fleuve important; lorsque survient la fonte d'un glacier ; lorsqu'un séisme isole en mer intérieure une partie des eaux d'un océan ; lorsque des individus se mettent en tête de creuser un immense trou et d'y acheminer les cours d'eau du bassinversant le plus proche; et pour toutes sortes d'autres raisons. Une fois en place, néanmoins, le lac développe des écosystèmes singuliers, et des relations non moins singulières avec son environnement : à tel point que les limnologues ont pu, à partir de ces régularités, constituer une discipline scientifique spécialisée. La démocratie est comme le lac : son comportement traduit des propriétés distinctives et une logique spécifique, ce qui n'empêche pas que chaque démocratie advient à sa manière, et retient dans son fonctionnement les traces de son histoire singulière.

Mais trêve de comparaison, avant qu'elle ne nous noie ! En bref, l'idée est la suivante : a priori, il n'y a absolument aucun motif de penser qu'un ensemble unique de facteurs produise et préserve la démocratie. Et cela nonobstant le fait que, au cours des cent dernières années, elle fut souvent nourrie de circonstances particulières ».

Charles Tilly, « Democracy Is a Lake », dans George Reid Andrews, Herrick Chapman (dir.), The Social Construction of Democracy, New York / Londres, New York University Press / Macmillan, 1995, p. 365-387 (ici cité d'après rééd. dans C. Tilly, Roads from past to future, Lanham, Rowman \& Littlefield, 1997, p. 209-210).

bien (a) les segments résultant du démantèlement de l'empire se dotent d'institutions régionales qui favorisent l'instauration d'une citoyenneté élargie, égalitaire, consultative et protectrice ; ou bien (b) la dissolution du gouvernement indirect, au bénéfice d'une relation de citoyenneté plus 
directe et uniforme, donne lieu à l'établissement de liens multiples entre le centre et les périphéries.

L'histoire a connu quelques exemples de la situation (a), mais, jusqu'à aujourd'hui, (b) demeure sans précédent. À l'échelle régionale, pourtant, la trajectoire (b) semble être une condition nécessaire de la démocratisation, au même titre que la subordination du pouvoir militaire au contrôle civil, la constitution de larges coalitions de classe appuyant l'action de l'État, et le déploiement d'un vaste système fiscal assurant la représentation des contribuables ${ }^{16}$. Faute d'une dissolution poussée du gouvernement indirect, les droits des citoyens demeurent fragiles et inégaux. Cette observation signale d'ailleurs la faille critique des programmes anarchistes ou capitalistes qui, de manière récurrente, prônent la décentralisation (ou le démantèlement total de l'État) comme voie d'accès à la démocratie : car les droits eux-mêmes, faute d'une instance [agency] qui les fasse respecter uniformément et effectivement, sont voués à disparaître ${ }^{17}$.

Un autre cas d'étude à retenir est celui du nationalisme. Ses éruptions sont-elles corrélées aux fins d'empire de manière étroite et générique ? La question importe, pour une part, en raison de l'accélération nationaliste provoquée par la désintégration de la Yougoslavie, de l'Union soviétique et du Pacte de Varsovie ; pour une part aussi, du fait des curieuses analogies apparues entre ces épisodes récents et le déclin des empires ottoman et austro-hongrois ; mais, surtout, cette question importe parce qu'elle signale une zone de désaccords potentiellement fructueux au sein des études sur le nationalisme. Une large gamme de travaux, sans être tous congruents entre eux, semblent d'accord pour conclure que le nationalisme devrait coïncider avec le déclin des empires. Ils arguent que toutes les nations développent, dans la mesure du possible, une résistance à la subordination ; que les empires sont, en fait, créateurs de nationalités ; et/ou que, dans un contexte de désintégration impériale, les puissances voisines poussent les sujets de l'empire au séparatisme ${ }^{18}$.

À quoi répliquent des analyses historicistes dont la logique causale paraît non moins contradictoire : le nationalisme, soulignent-elles, est apparu à une période relativement récente, suite à un processus historique spécifique ${ }^{19}$, et demeure qui plus est susceptible de disparaître eu égard à la modification des conditions historiques. Eric Hobsbawm et moi-même ne sommes d'ailleurs pas les derniers à avoir fait valoir cette veine d'interprétation du nationalisme $\mathrm{y}$ prédominent les définitions de la nation promues par l'État, imposées à l'ordinaire des populations ; 
réciproquement, la reconnaissance ultérieure de l'auto-détermination nationale y fait office de pierre de touche pour la reconnaissance des États nouvellement fondés ${ }^{20}$. D'où une confrontation prometteuse entre tenants d'une analyse générique et partisans de positions historicistes — en quelque sorte un match entre Anthony Smith et Rogers Brubaker ${ }^{21}$ ! - confrontation qui pourrait permettre de démêler, outre l'intrigue embrouillée du nationalisme, l'écheveau des fins d'empire.

\author{
Traduit de l'anglais (États-Unis) par Marc Aymes
}

1. Lewis Mumford, La Cité à travers l'histoire (trad. de l'anglais par Guy et Gérard Durand, ici avec révisions), Paris, Seuil, 1964 [1961], p. 310-311.

2. Alexander J. Motyl, « From Imperial Decay to Imperial Collapse : The Fall of the Soviet Empire in Comparative Perspective »,dans Richard J. Rudolph, David F. Good (dir.), Nationalism and Empire. The Habsburg Empire and the Soviet Union, New York, St. Martin's Press, 1992. (Motyl a depuis publié Imperial Ends : The Decay, Collapse, and Revival of Empire; New York : Columbia University Press, 2001 - titre qui n'est pas sans faire écho à celui du présent article, N.d.T.)

3. Voir Martin van Creveld, Technology and War from 2000 B.C. to the Present (New York, Free Press, 1989) ; id.., The Transformation of War, New York, Free Press, 1991 ; Helen Fein, « Accounting for Genocide after 1945 : Theories and Some Findings », International Journal on Group Rights, 1 (1993), p. 79-106 ; Ted Robert Gurr, Minorities at Risk. A Global View of Ethnopolitical Conflicts, Washington, D.C., U.S. Institute of Peace Press, 1993 ; et Ted Robert Gurr, Barbara Harff, «Victims of the State : Genocides, Politicides, and Group Repression since 1945 », International Review of Victimology, 1 (1989), p. 23-41.

4. Voir Stephen Graubard (dir.), « Reconstructing Nations and States », Daedalus 122 (1993) ; id.., « Europe Through a Glass Darkly », Daedalus 123 (1994) ; id., «What Future for the State? », Daedalus 124 (1995) ; Hugh Gusterson, « Realism and the International Order after the Cold War », Social Research 60 (1993), p. 279-300 ; Istvan Hont, « The Permanent Crisis of a Divided Mankind : "Contemporary Crisis of the Nation State" in Historical Perspective », Political Studies 42 (1994), p. 166-231; John Gerard Ruggie, «Territoriality and Beyond : Problematizing Modernity in International Relations », International Organization 47 (1993), p. 139-174 ; Sidney Tarrow, « La mondialisation des conflits : encore un siècle de rébellion? », Études internationales 24 (1993), p. 513-532 ; Janice E. Thomson, «State Sovereignty in International Relations : Bridging the Gap Between Theory and Empirical Research », International Studies Quaterly 39 (1995), p. 213-234 ; et Charles Tilly, « Globalization Threatens Labor's Rights », International Labor and Working Class History 47 (1995), p. 1-23.

5. Gabriel Ardant, Théorie sociologique de l'impôt, Paris, SEVPEN, 1965, 2 vol. ; John L. Campbell, «The State and Fiscal Sociology », Annual Review of Sociology 19 (1993), p. 163-185.

6. Kung-Chuan Hsiao, Rural China : Imperial Control in the Nineteenth Century, Seattle, Universitv of Washington Press, 1960 ; Stuart Schram (dir.), The Scope of State Power in China (1985) et Foundations and Limits of State Power in China (1987) (volumes publiés pour la Fondation européenne de la science par la School of Oriental and African Studies, University of London, et par Chinese Universitv Press of Hong Kong ; Vivienne Shue, The Reach of the State: Sketches of the Chinese Body Politic, Stanford, Stanford University Press, 1988 ; William G. Skinner, « Marketing and Social Structure in Rural China », Journal of Asian Studies 24 (1964), p. 3-43; id., « The Structure of Chinese History », Journal of Asian 


\section{Labyrinthe, $n^{\circ} 35$}

Studies 44 (1985), p. 271-292 ; Frederic Wakeman, Strangers at the Gate : Social Disorder in South China, 1839-1861, Berkeley, University of California Press, 1966 ; id., The Great Enterprise : The Manchu Reconstruction of Imperial Order in Seventeenth-Century China, Berkeley, University of California Press, 1985 ; Joseph B. R. Whitney, China : Area, Administration, and Nation Building, Research Paper 123 (Chicago, Department of Geography, University of Chicago, 1970) ; Pierre-Étienne Will, «Chine moderne et sinologie », Annales. Histoire, Sciences Sociales 49 (1994), p. 7-26 ; Pierre-Étienne Will et R. Bing Wong, Nourish the People: The State Civilian Granary System in China, 1650-1850, Ann Arbor, University of Michigan Press, 1991 ; R. Bin Wong, « Les Émeutes de subsistances en Chine et en Europe Occidentale », Annales. Économies, Sociétés, Civilisations 38 (1983), p. 234-258.

7. Joseph A. Tainter, The Collapse of Complex Societies, Cambridge, Cambridge University Press, 1988.

8. Voir par exemple Thomas A. Brady, Jr., Turning Swiss : Cities and Empire, 1450-1550, Cambridge, Cambridge University Press, 1985 ; David Strang, « From Dependency to Sovereignty : An Event History Analysis of Decolonization, 1870-1987 », Americain Sociological Review 55 (1990), p. 846-860 ; et id., « Global Patterns of Decolonization, 1500-1987 », International Studies Quaterly 35 (1991), p. 429-454. 9. William H. Beik, Absolutism and Society in Seventeenth-Century France, Cambridge, Cambridge University Press, 1985 ; Nicholas Henshall, The Myth of Absolutism : Change and Continuity in Early Modern European Monarchy, Londres, Longman, 1992 ; Sharon Kettering, « Brokerage at the Court of Louis XIV », Historical Journal 36 (1993), p. 69-87 ; Richard Lachmann et Julia Adams, « Absolutism's Antinomies : Class Formation, State Fiscal Structures and the Origins of the French Revolution », Political Power and Social Theory 7 (1988), p. 135-175.

10. Isser Woloch, The New Regime : Transformations of the French Civic Order, 1789-1820s, New York, Norton, 1994.

11. En français dans le texte. Une précision aussi concernant la théorie du principal-agent : pièce maîtresse des théories de l'économie industrielle, elle traite des difficultés qui surviennent dans une organisation suite à une asymétrie d'information, c'est-à-dire lorsque l'action d'un acteur, le « principal », dépend des démarches d'un autre acteur, «l'agent », sur lequel le principal est imparfaitement informé. [N.d.T.]

12. S. N. Eisenstadt, The Political Systems of Empires : The Rise and Fall of the Historical Bureaucratic Societies, New York, Free Press of Glencoe, 1963 ; cf. David E. M. Sappington, « Incentives in PrincipalAgent Relationships », Journal of Economic Perspectives 5 (1991), p. 45-66.

13. John H. Pryor, Geography, Technology, and War : Studies in the Maritime History of the Mediterranean, 649-1571, Cambridge, Cambridge University Press, 1988.

14. J. H. Elliott, Imperial Spain, 1469-1716, Londres, Edward Arnold, 1963 ; Richard Herr, Rural Change and Royal Finances in Spain at the End of the Old Regime, Berkeley, University of California Press, 1989 ; Alberto Tenenti, Piracy and the Decline of Venice, 1580-1615, Berkeley, University of California Press, 1967 ; et Janice E. Thomson, Mercenaries, Pirates, and Sovereigns : State-Building and Extraterritorial Violence in Early Modern Europe, Princeton, Princeton University Press, 1994.

15. Anthony Arblaster, Democracy, Minneapolis, University of Minnesota Press, 1987 ; Ross E. Burkhart et Michael S. Lewis-Beck, « Comparative Democracy : The Economic Development Thesis », American Political Science Review 88 (1994), p. 903-910 ; Robert Dahl, Democracy and Its Critics, New Haven, Yale University Press, 1989 ; Larry Diamond et Gary Marks (dir.), « Comparative Perspectives on Democracy : Essays in Honor of Seymour Martin Lipset », numéro spécial de l'American Behavioral Scientist 35 (1992) ; Giuseppe Di Palma, To Craft Democracies : An Essay on Democratic Transitions, Berkeley, University of California Press, 1990 ; Eva Etzioni-Halevy, The Elite Connection : Problems and Potential of Western Democracy, Cambridge, Polity Press, 1993 ; Steven M. Fish, Democracy from Scratch : Opposition and Regime in the New Russian Revolution, Princeton, Princeton University Press, 1995 ; David Held, Models of Democracy, Stanford, Stanford University Press, 1987 ; Alex Inkeles (dir.), On Measuring Democracy, Its Consequences and Concomitants, New Brunswick, Transaction, 1991 ; Alberto Melucci, «Liberation or Meaning ? Social Movements, Culture and Democracy », Development and Change 23 (1992), p. 43-77 ; Edward N. Muller et Mitchell A. Seligson, « Civic 


\section{Comment les empires touchent à leur fin}

Culture and Democracy : The Question of Causal Relationships », Americain Political Science Review 88 (1994), p. 635-652 ; Guillermo O'Donnell et Philippe C. Shmitter, Transitions from Authoritarian Rule : Prospects for Democracy, Baltimore, The Johns Hopkins University Press, 1986 ; Robert D. Putnam, Making Democracy Work : Civic Traditions in Modern Italy, Princeton, Princeton University Press, 1993 ; Dietrich Rueschemeyer, Evelyne Huber Stephens et John D. Stephens, Capitalist Development and Democracy, Chicago, University of Chicago Press, 1992 ; Philippe C. Schmitter et Terry Lynn Karl, « What Democracy Is... and Is Not », Journal of Democracy 2 (1991), p. 77-88.

16. Charles Tilly, «Democracy Is a Lake », dans George Reid Andrews, Herrick Chapman (dir.), The Social Construction of Democracy, New York / Londres, New York University Press / Macmillan, 1995), p. 365-387.

17. Charles Tilly, « Globalization Threatens Labor's Rights », International Labor and Working Class History 47 (1995), p. 1-23.

18. Walker Connor, «Ethnonationalism », dans Myron Weiner, Samuel P. Huntington (dir.), Understanding Political Development, Boston, Little \& Brown, 1987 ; Karl Deutsch, Nationalism and social communication, Cambridge, MIT Press, 1966 ; Donald Horowitz, Ethnic Groups in conflict, Berkeley, University of California Press, 1985 ; et Anthony D. Smith, The Ethnic Revival, Cambridge, Cambridge University Press, 1981.

19. John A. Armstrong, Nations Before Nationalism, Chapel Hill, University of North Carolina Press, 1982 ; Benedict Anderson, Imagined Communities : Reflections on the Origin and Spread of Nationalism, Londres, Verso, 1991 ; Ernest Gellner, Nations and Nationalism, Ithaca, Cornell University Press, 1983 ; Liah Greenfeld, Nationalism : Five Roads to Modernity, Cambridge, Harvard University Press, 1992 ; Miroslav Hroch, Social Preconditions of National Revival in Europe : A Comparative Analysis of the Social Composition of Patriotic Groups Among the Smaller European Nations, Cambridge, Cambridge University Press, 1985.

20. E. J. Hobsbawm, Nations and Nationalism Since 1789 : Programme, Myth, Reality, Cambridge, Harvard University Press, 1990 ; Charles Tilly, « States and Nationalism in Europe 1492-1992 », Theory and Society 23 (1994), p. 131-146.

21. De ce dernier, citons notamment ici Nationalism Reframed : Nationhood and the National Question in the New Europe, Cambridge, Cambridge University Press, 1996. [N.d.T.] 